\title{
Use of Targeted High-Throughput Sequencing for Genetic Classification of Patients with Bleeding Diathesis and Suspected Platelet Disorder
}

\author{
Oliver Andres ${ }^{1}$ Eva-Maria König ${ }^{2}$ Karina Althaus ${ }^{3,4}$ Tamam Bakchoul $^{3,4}$ Peter Bugert ${ }^{5}$ \\ Stefan Eber ${ }^{6}$ Ralf Knöfler ${ }^{7}$ Erdmute Kunstmann ${ }^{2}$ Georgi Manukjan ${ }^{8}$ Oliver Meyer $^{9}$ \\ Gabriele Strauß ${ }^{10}$ Werner Streif ${ }^{11}$ Thomas Thiele ${ }^{4}$ Verena Wiegering ${ }^{1}$ Eva Klopocki ${ }^{2}$ \\ Harald Schulze ${ }^{8}$ on Behalf of the THROMKIDplus Study Group of the Society of Paediatric Oncology \\ Haematology (Gesellschaft für Pädiatrische Onkologie und Hämatologie, GPOH) and the Society of \\ Thrombosis Haemostasis Research (Gesellschaft für Thrombose- und Hämostaseforschung, GTH)
}

\footnotetext{
1 University Children's Hospital, University of Würzburg, Würzburg, Germany

2 Institute of Human Genetics, University of Würzburg, Würzburg, Germany

${ }^{3}$ Centre for Clinical Transfusion Medicine, University Hospital of Tübingen, Tübingen, Germany

${ }^{4}$ Institute for Transfusion Medicine, University of Greifswald, Greifswald, Germany

${ }^{5}$ DRK-Blutspendedienst Baden-Württemberg-Hessen, Institute for Transfusion Medicine and Immunology, Heidelberg University, Mannheim, Germany

6University Children's Hospital, Technical University Munich, Munich, Germany

${ }^{7}$ Department of Pediatrics, Carl Gustav Carus University Hospital, Dresden, Germany
}

\author{
Address for correspondence Prof. Dr. rer. nat. Harald Schulze, \\ Institute of Experimental Biomedicine, Chair I, Haus D15, Josef- \\ Schneider-Straße 2, 97080 Würzburg, Germany \\ (e-mail: harald.schulze@uni-wuerzburg.de). \\ 8 Institute of Experimental Biomedicine, University Hospital \\ Würzburg, Würzburg, Germany \\ ${ }^{9}$ Institute for Transfusion Medicine, Charité-Universitätsmedizin \\ Berlin, Berlin, Germany \\ ${ }^{10}$ Department for Pediatric Oncology and Hematology, HELIOS \\ Klinikum Berlin-Buch, Berlin, Germany \\ ${ }^{11}$ Department of Pediatrics, Medical University Innsbruck, Innsbruck, \\ Austria
}

TH Open 2018;2:e445-e454.

\section{Abstract \\ Keywords \\ - next-generation sequencing \\ - molecular genetics \\ - thrombocytopenia \\ - thrombocytopathy \\ - platelet function disorder}

Inherited platelet disorders (IPD) form a rare and heterogeneous disease entity that is present in about $8 \%$ of patients with non-acquired bleeding diathesis. Identification of the defective cellular pathway is an important criterion for stratifying the patient's individual risk profile and for choosing personalized therapeutic options. While costs of high-throughput sequencing technologies have rapidly declined over the last decade, molecular genetic diagnosis of bleeding and platelet disorders is getting more and more suitable within the diagnostic algorithms. In this study, we developed, verified, and evaluated a targeted, panel-based next-generation sequencing approach comprising 59 genes associated with IPD for a cohort of 38 patients with a history of recurrent bleeding episodes and functionally suspected, but so far genetically undefined IPD. DNA samples from five patients with genetically defined IPD with disease-causing variants in WAS, RBM8A, FERMT3, P2YR12, and MYH9 served as controls during the validation process. In $40 \%$ of 35 patients analyzed, we were able to finally detect 15 variants, eight of which were novel, in 11 genes, ACTN1, AP3B1, GFI1B, HPS1, HPS4, HPS6, MPL, MYH9, TBXA2R, TPM4, and TUBB1, and classified them according to current guidelines. Apart from seven variants of uncertain significance in $11 \%$ of patients, nine received

July 17, 2018

accepted after revision

November 19, 2018
DOI https://doi.org/

10.1055/s-0038-1676813. ISSN 2512-9465. (c) 2018 Georg Thieme Verlag KG Stuttgart · New York
License terms

(c) (1) 
variants were classified as likely pathogenic or pathogenic providing a molecular diagnosis for $26 \%$ of patients. This report also emphasizes on potentials and pitfalls of this tool and prospectively proposes its rational implementation within the diagnostic algorithms of IPD.

\section{Introduction}

Inherited platelet disorders (IPD) form a rare and heterogeneous disease entity that is present in about $8 \%$ of patients with non-acquired bleeding diathesis and characteristically associated with mucocutaneous bleeding episodes of variable intensity that may culminate in life-threatening hemorrhage during or after invasive procedures. ${ }^{1-3}$ Impaired transcription regulation, receptor signaling, cytoskeleton formation, granule composition, or trafficking in megakaryocytes or platelets may result in a reduced circulating platelet count, platelets failing to fulfil their hemostatic function, or even a combined quantitative and qualitative platelet defect. ${ }^{1}$ Identification of the defective cellular pathway is an important criterion for stratifying the patient's individual risk profile and for choosing personalized therapeutic options. ${ }^{4}$ In addition, precise determination of the gene or pathway involved is substantial for trustworthy genetic counselling of patients and their relatives with respect to the mode of inheritance, recurrence risk, and long-term prognosis, especially in disorders with different inheritance patterns, for example, autosomal dominant Bernard-Soulier syndrome type A2 (OMIM 153670) or severe gray platelet-like disease caused by recently described novel autosomal recessive GFI1B variants. ${ }^{5-7}$

To reach these aims, various diagnostic tools to assess platelet biogenesis and function have been developed over the last decades. ${ }^{8}$ Several national and international guidelines have been elaborated to standardize diagnostic processes and laboratory assays. ${ }^{9-11}$ A recent worldwide laboratory survey of the International Society on Thrombosis and Haemostasis (ISTH), however, revealed that, even if a broad range of analytical methods is applied, the exact underlying defect cannot be identified in more than onethird of patients with confirmed platelet function abnormality. ${ }^{12}$ Since knowledge about genes, that are involved in either quantitative or qualitative platelet disorders, has increased rapidly and costs of high-throughput sequencing (HTS) technologies have declined simultaneously over the last decade, molecular genetic diagnosis of bleeding and platelet disorders (BPDs) is getting more and more valuable in diagnostic algorithms. ${ }^{13-15}$

Several initiatives, primarily from centers in the United Kingdom, have selected and adapted HTS techniques to accelerate the time to diagnose IPD or to identify novel genes or variants involved in the pathogenesis of these rare diseases. The international collaborative ThromboGenomics and BRIDGE-BPD projects have provided a significant impact on research in the field of BPD. While the ThromboGenomics targeted sequencing platform comprises a limited number of initially 63, currently 79 genes with a characterized role in bleeding, thrombotic, or platelet disorders, the BRIDGE-BPD consortium chose a whole exome sequencing (WES) approach that is linked to a standardized phenotype classification, using the human phenotype ontology (HPO), to identify novel genes eliciting a certain phenotype pattern. ${ }^{16-19}$ The United Kingdom Genotyping and Phenotyping of Platelets (GAPP) study has specifically focused on platelets and has identified several novel variants and genes by using HTS techniques. ${ }^{20-22}$ Just recently, a Scandinavian collaborative group and a Spanish group each published their experiences of diagnosing and classifying BPD by targeted HTS methods and found significant variants in 22 or $68 \%$ of the investigated patients, respectively. ${ }^{23,24}$

In parallel, members of the German, Austrian, and Swiss THROMKIDplus study group developed, verified, and evaluated a targeted, panel-based next-generation sequencing (NGS) approach for a cohort of 38 patients with a history of recurrent bleeding episodes and functionally suspected, but so far genetically undefined IPD. All detected variants were classified as pathogenic (class 5), likely pathogenic (class 4 ), uncertain significance (class 3 ), likely benign (class 2 ), or benign (class 1 ). This report presents the results of this study and also emphasizes on potentials and pitfalls of this tool and prospectively proposes its rational implementation within the diagnostic algorithms of IPD.

\section{Materials and Methods}

\section{Subjects}

Thirty-eight unrelated individuals between 1 month and 66 years of age ( 26 children or adolescents and 12 adults) with suspected IPD were included into the study between January 2015 and January 2016 (-Table 1). Each patient had been evaluated in specialized centers in Germany and Austria between June 2007 and January 2016 due to a mild to severe bleeding diathesis according to current guidelines and considered to have a familial, syndromic, or functionally diagnosed platelet disorder with onset in childhood or adolescence. ${ }^{10}$ Among these 38 patients whose diagnoses were not genetically defined prior to enrolment, 18 patients (47\%) suffered from isolated thrombocytopenia, 16 patients (42\%) from a platelet function disorder, and four patients from a combined defect (11\%). Among them were four patients highly suggestive to have Hermansky-Pudlak syndrome (HPS) due to impaired platelet function and oculocutaneous albinism. Full blood count and blood smears stained according to May-Grünwald-Giemsa or Pappenheim showed macrothrombocytopenia in six patients (27\% of cases with thrombocytopenia). Immunofluorescence microscopy for 
Genetic Classification of Patients with Bleeding Diathesis and Suspected Platelet Disorder Andres et al. e447

\begin{tabular}{|c|c|c|c|c|c|c|c|c|c|c|c|c|c|c|c|c|c|c|}
\hline 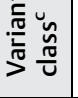 & $\nabla$ & $\sim$ & & & & & $m$ & & 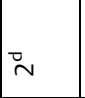 & in & & $\nabla$ & & $\nabla$ & & $m$ & & \\
\hline 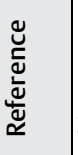 & I & 1 & & & & & 1 & & $\begin{array}{l}9 \\
\text { a } \\
\text { d. } \\
m\end{array}$ & & & li & & 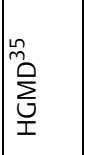 & & 1 & & \\
\hline 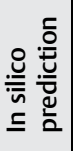 & 1 & $\begin{array}{l}0 \\
\dot{v} \\
\Sigma\end{array}$ & & & & & $\begin{array}{l}a \\
v i \\
\dot{\Sigma}\end{array}$ & & $\begin{array}{l}0 \\
v \\
\sum \\
\dot{\Sigma} \\
\omega\end{array}$ & 1 & & 1 & & $\Sigma$ & & $\mid \begin{array}{l}\sum \\
\text { J }\end{array}$ & & \\
\hline 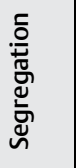 & 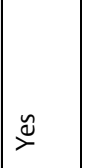 & $\stackrel{0}{z}$ & & & & & $\begin{array}{l}\dot{I} \\
\dot{c}\end{array}$ & & $\begin{array}{l}\dot{\dot{x}} \\
\dot{c}\end{array}$ & $\stackrel{\Perp}{\succ}$ & & $\begin{array}{l}\dot{J} \\
\dot{\tau}\end{array}$ & & $\dot{\dot{C}}$ & & $\begin{array}{l}\dot{\dot{x}} \\
\dot{c}\end{array}$ & & \\
\hline 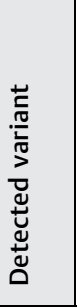 & 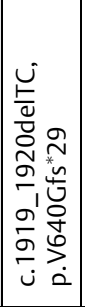 & 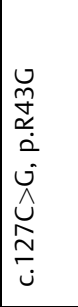 & & & & & 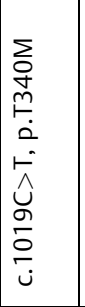 & & 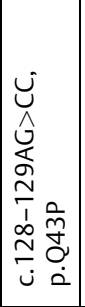 & 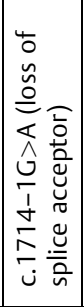 & & 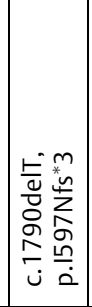 & & 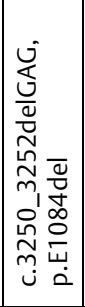 & & 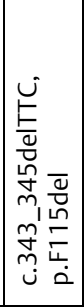 & & \\
\hline 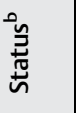 & $\begin{array}{l}E \\
\\
\end{array}$ & $\stackrel{ \pm}{\Phi}$ & & & & & 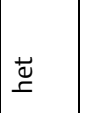 & & 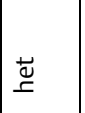 & $\begin{array}{l}E \\
\\
\end{array}$ & & E & & 苟 & & 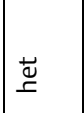 & & \\
\hline ֻัّ & 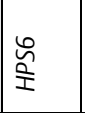 & 窝怘 & & & & & 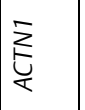 & & $\begin{array}{l}\bar{\alpha} \\
\underline{p} \\
\stackrel{p}{R}\end{array}$ & 苾 & & $\begin{array}{l}\bar{m} \\
\text { ֻे }\end{array}$ & & 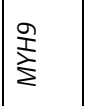 & & 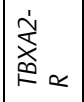 & & \\
\hline 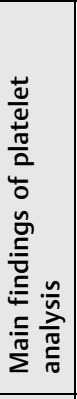 & 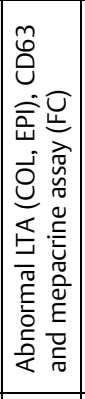 & \begin{tabular}{|l} 
\\
\\
\\
\\
$\dot{r}$ \\
$\dot{\check{L}}$ \\
$\dot{0}$
\end{tabular} & 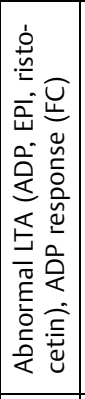 & $\begin{array}{l} \\
\\
\\
\\
\dot{r} \\
\dot{\dot{x}} \\
\dot{0}\end{array}$ & \begin{tabular}{|l}
$\dot{r}$ \\
$\dot{c}$ \\
$\dot{\sigma}$
\end{tabular} & 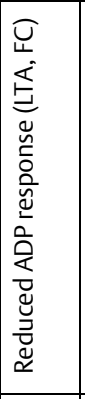 & $\begin{array}{l}\dot{\sigma} \\
\dot{5} \\
\dot{\sigma}\end{array}$ & 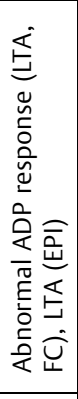 & 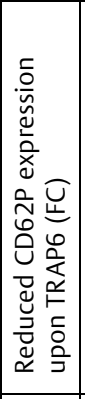 & 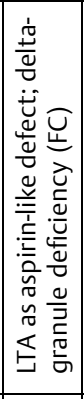 & $\begin{array}{l} \\
\\
\\
\\
\dot{0} \\
\dot{\check{0}} \\
\dot{0}\end{array}$ & 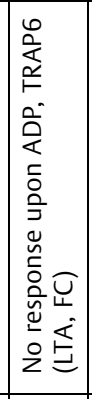 & 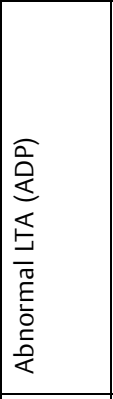 & $\begin{array}{l}\dot{r} \\
\dot{5} \\
\dot{0}\end{array}$ & 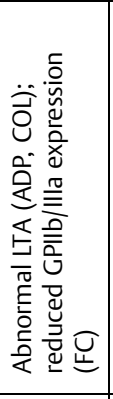 & \begin{tabular}{|l} 
\\
\\
\\
$\dot{r}$ \\
$\dot{\check{j}}$ \\
$\dot{0}$
\end{tabular} & 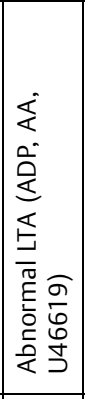 & 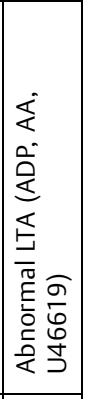 \\
\hline 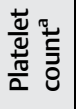 & $\stackrel{ }{N}$ & 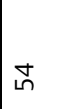 & $\Sigma$ & $m$ & $\wedge$ & $\dot{\ddot{d}}$ & $\begin{array}{l}\dot{ர} \\
\dot{\check{C}}\end{array}$ & $\stackrel{\oslash}{\sim}$ & 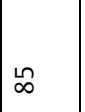 & 㸃 & $\stackrel{\cong}{\circ}$ & $\stackrel{\dot{r}}{\dot{c}}$ & $\stackrel{\infty}{\sim}$ & $\stackrel{\llcorner}{\mp}$ & $\stackrel{\infty}{\leftarrow}$ & $\stackrel{\grave{N}}{\simeq}$ & $\stackrel{\circ}{\circ}$ & 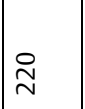 \\
\hline 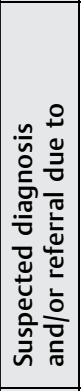 & 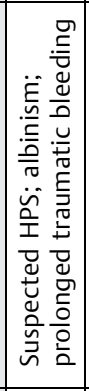 & 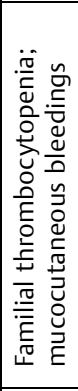 & 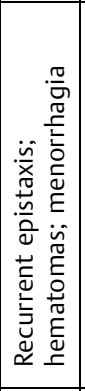 & 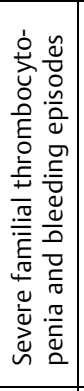 & 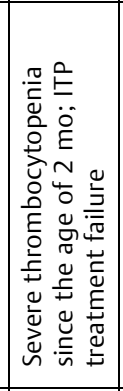 & 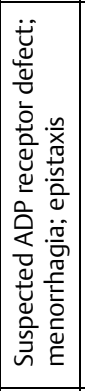 & 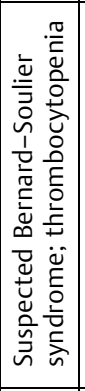 & 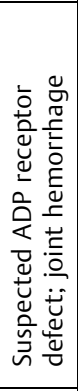 & 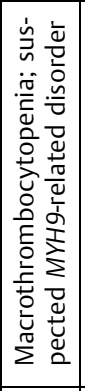 & 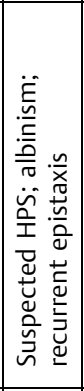 & 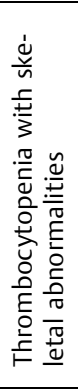 & 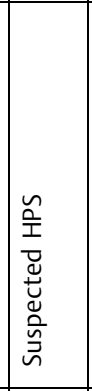 & 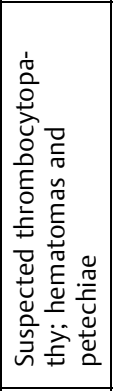 & 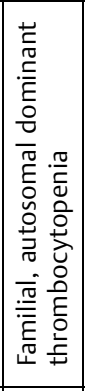 & 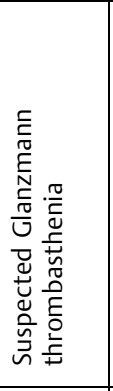 & 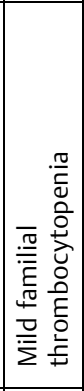 & 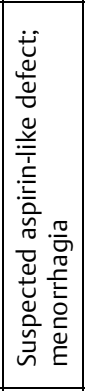 & 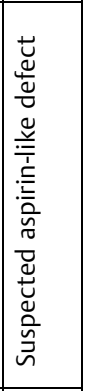 \\
\hline ঐ & $E$ & $E$ & + & $E$ & 4 & $E$ & 4 & $E$ & + & + & $E$ & + & $E$ & $E$ & + & $E$ & + & + \\
\hline 芩 & $\stackrel{2}{2}$ & 畩 & $\stackrel{2}{=}$ & $\vec{m}$ & $\vec{a}$ & $\begin{array}{l}\stackrel{\circ}{E} \\
\stackrel{\leftrightarrow}{\curvearrowleft}\end{array}$ & $\vec{\theta}$ & $\overrightarrow{ \pm}$ & $\vec{o}$ & $\overrightarrow{0}$ & $\begin{array}{l}\lambda \\
\Sigma\end{array}$ & $\underset{\Xi}{\Delta}$ & $\vec{m}$ & $\stackrel{\infty}{\longrightarrow}$ & $\begin{array}{l}\stackrel{\circ}{E} \\
\stackrel{\text { I }}{ \pm}\end{array}$ & Oे & $\stackrel{\infty}{\infty}$ & 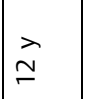 \\
\hline ֶّ & - & $\sim$ & $m$ & $\nabla$ & n & 0 & $\wedge$ & $\infty$ & $a$ & 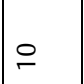 & $=$ & $\simeq$ & $m$ & $\stackrel{\Delta}{ \pm}$ & $\stackrel{\text { ㅇ }}{ }$ & $\stackrel{\varphi}{\circ}$ & $\neq$ & $\stackrel{\infty}{\leftarrow}$ \\
\hline
\end{tabular}




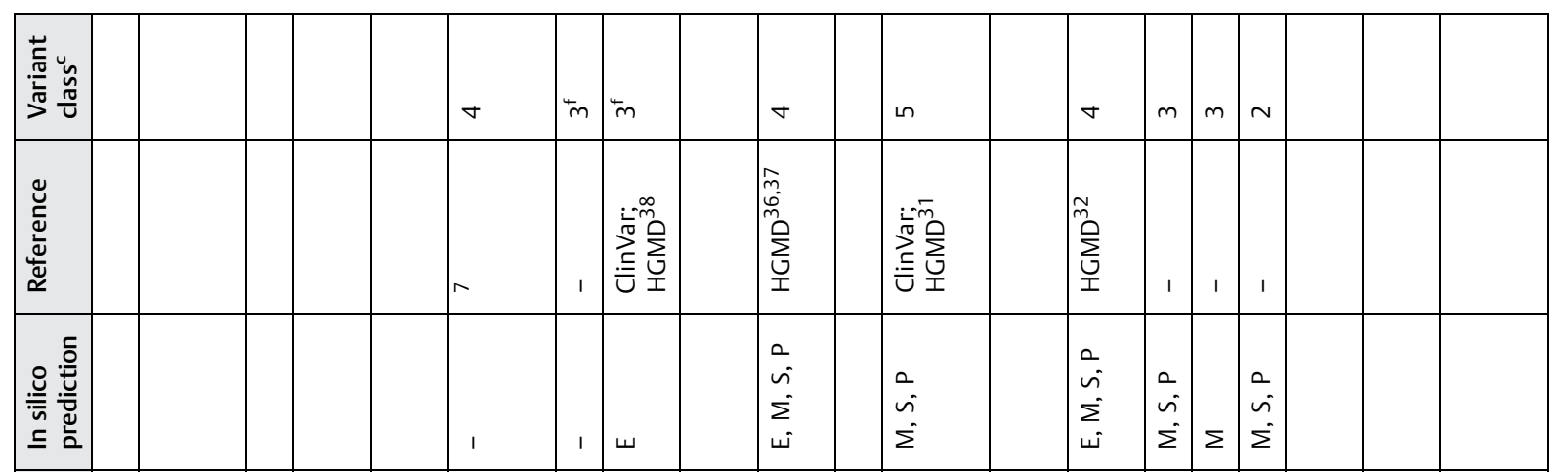

듬

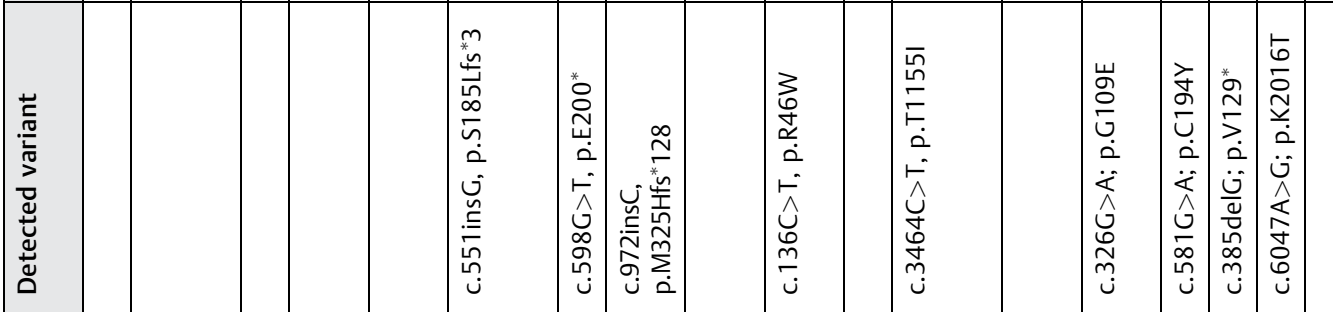

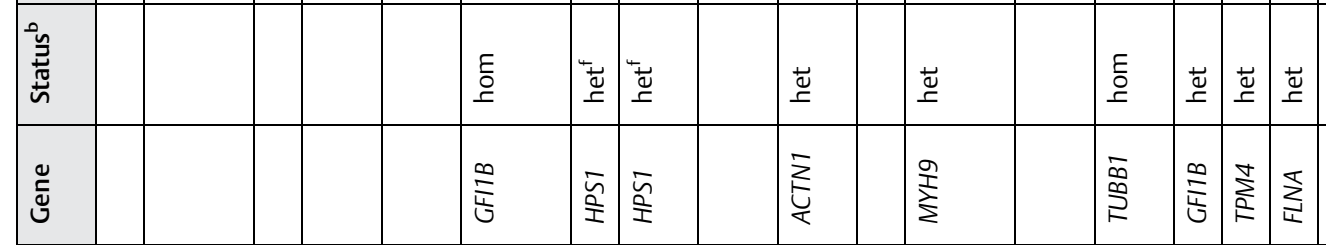

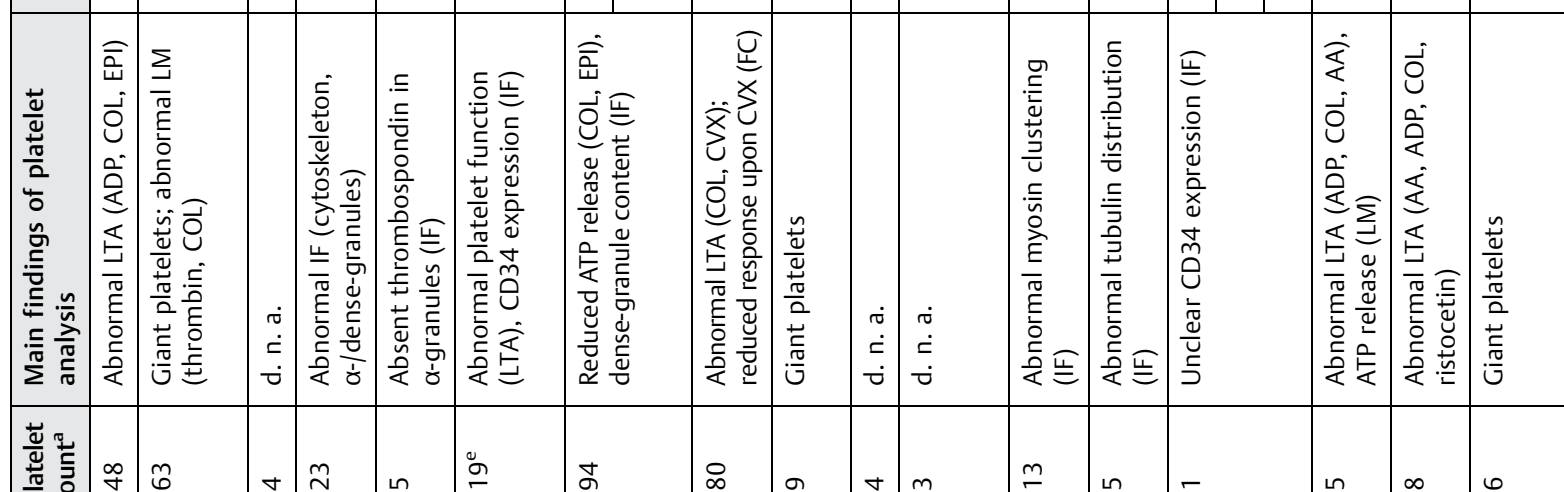

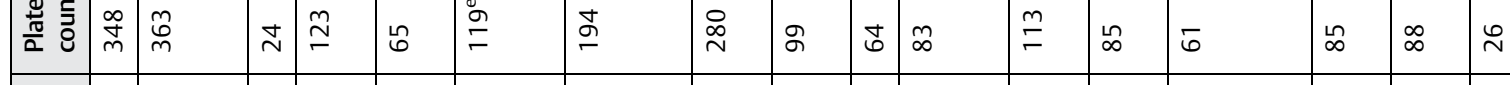

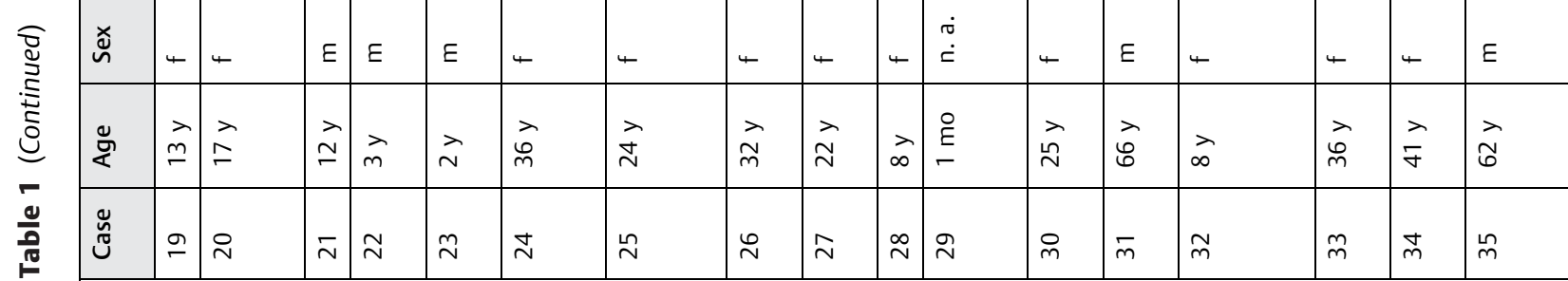




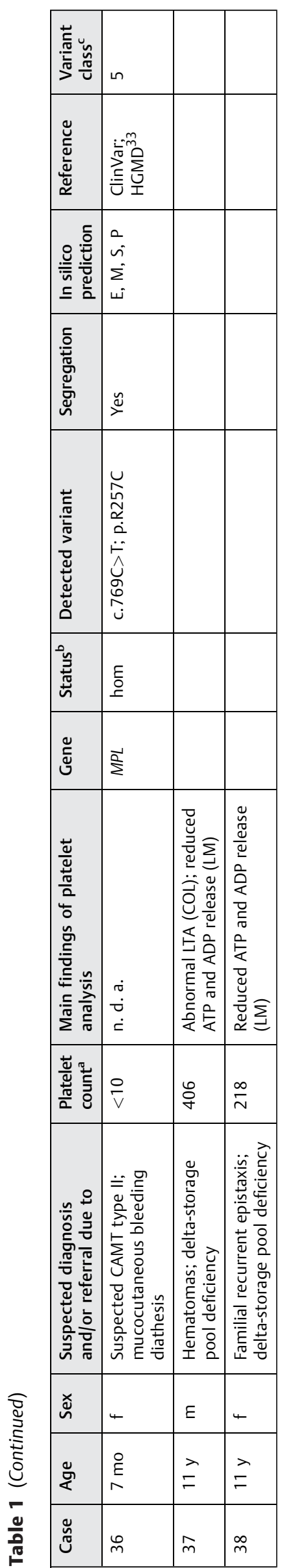

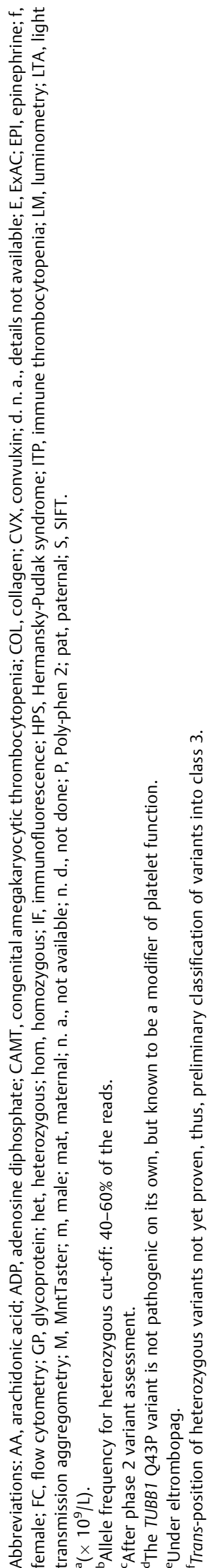

nonmuscle myosin heavy chain Ila expression was performed to confirm or rule out MYH9-associated disorders in indicated cases. ${ }^{10,25}$ In the group of platelet function disorders, blood samples were analyzed for plasmatic clotting and von Willebrand factors, Born aggregometry (basically using ADP, epinephrine, collagen, and ristocetin as agonists), and, in some cases, lumino-aggregometry and flow cytometry (mainly to assess Glanzmann thrombasthenia or Bernard-Soulier syndrome) as designated. ${ }^{10,26}$ The patient cohort typically reflects various reasons for referral, a broad distribution of treating centers, and a distinct access of even specialized centers to extended diagnostic methods and, thus, provides a rather heterogeneous list of available diagnostic results for this cohort (-Table $\mathbf{1}$ ).

For validation of the targeted, panel-based NGS approach, genomic DNA from five patients between 17 days and 14 years of age with functionally characterized and genetically defined IPD served as controls: adenosine diphosphate (ADP) receptor defect, leukocyte-adhesion deficiency type $\mathrm{III},{ }^{27}$ MYH9-related macrothrombocytopenia, thrombocytopenia-absent radius (TAR) syndrome, and Wiskott-Aldrich syndrome, respectively (-Supplementary Table S1).

\section{Gene Panel Design}

The gene panel was based on causes of quantitative or qualitative IPD that had been published by the time of its compilation in December 2014 and designed using Illumina DesignStudio software (Illumina, San Diego, California, United States). ${ }^{4,10,28}$ In contrast to the ThromboGenomics platform or the panel of the Scandinavian collaborative group, genes associated with deficient clotting factors or each type of von Willebrand disease were not taken into consideration, as plasmatic deficiencies are excluded by comprehensive laboratory analysis according to the current underlying guidelines. ${ }^{10,17,23}$ For most of the genes, the panel design was restricted to coding sequences. In case of ANKRD26 and $R B M 8 A$, which are reported to have variants in the noncoding 5 ' untranslated region (5'UTR) or in introns, the design included these noncoding regions. The panel design was supplemented with candidate genes or predisposing genes known from animal models finally resulting in a panel covering 59 genes (-Supplementary Table S1).

\section{Sequencing Procedure and Data Processing}

Genomic DNA was isolated from peripheral blood samples of 43 individuals and subjected to targeted, panel-based NGS. Of these 43 individuals, 38 patients had an unknown molecular etiology and 5 patients had well-characterized variants for the validation process (-Fig. 1). Target enrichment was performed by Nextera Rapid Capture Enrichment and subsequent sequencing on the MiSeq platform; the MiSeq Reporter was used for the alignment of the sequencing data (Illumina). Three datasets that failed quality control were excluded from further processing and analysis (-Fig. 1). The alignments and copy number variations (CNV) of RBM8A were analyzed by the GensearchNGS software (version 1.6.58; PhenoSystems SA, Wallonia, Belgium). Alamut Software Suite (version 2.7_1; Interactive Biosoftware, Rouen, France) served as a 


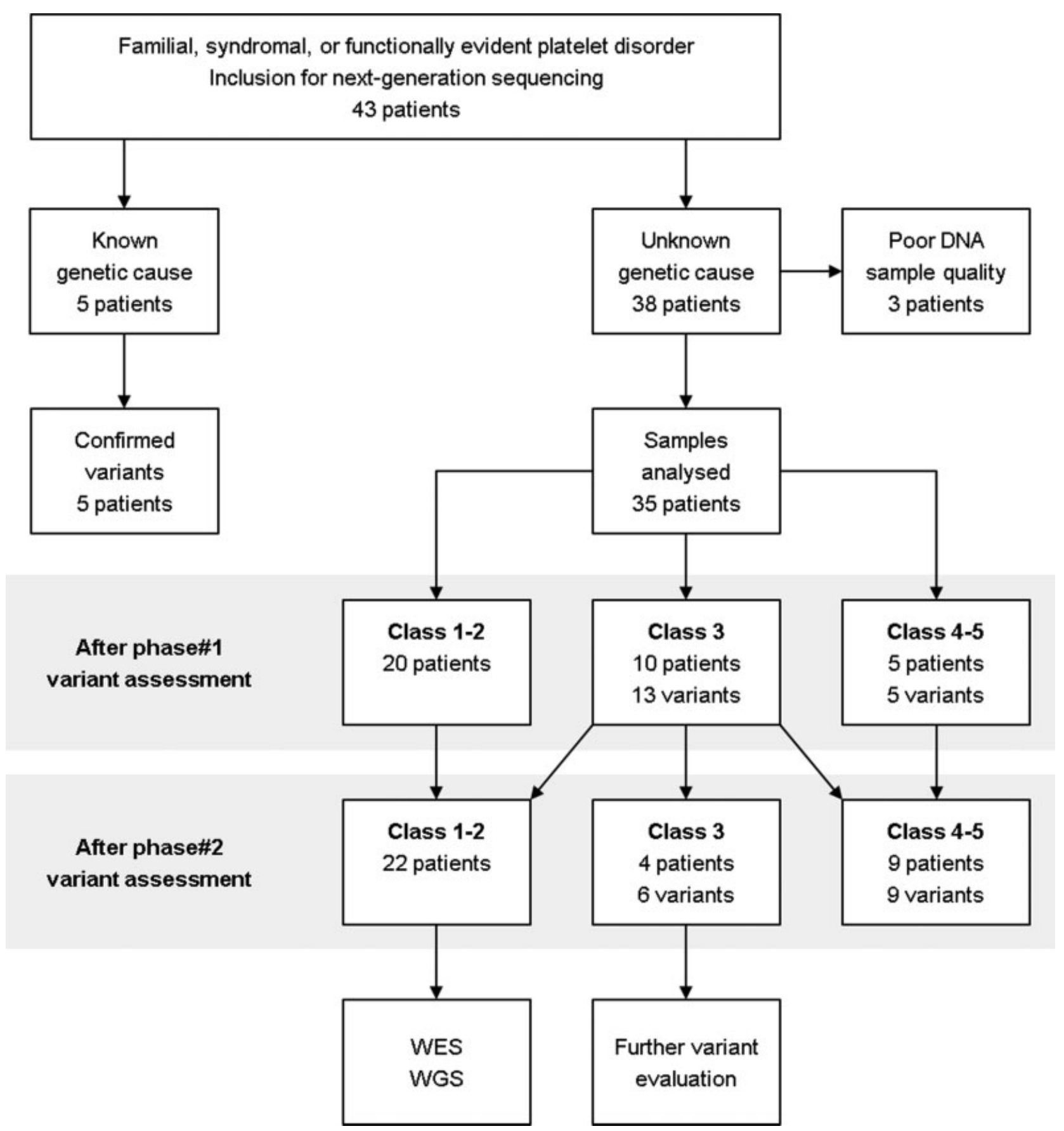

Fig. 1 Flow chart of analysis procedure and variant assessment. DNA samples of 43 individuals were processed by targeted, panel-based nextgeneration sequencing (absolute number of patients). In the group of patients with unknown platelet defect, variants were initially classified using in silico filtering programs and gene databases (phase 1 variant assessment). In a second step, class 3 variants (variants of uncertain significance) were assessed with respect to clinical phenotype, segregation analysis, literature, in vitro data, and animal models (phase 2 variant assessment). DNA, deoxyribonucleic acid; WES, whole exome sequencing; WGS, whole genome sequencing.

visualization tool. For variant identification, filters were set as follows: minor allele frequency (MAF) less than $1 \%$, balance of forward and reverse reads greater than 0 , and read count greater than 20. Nomenclature has been applied according to Human Genome Variation Society (HGVS; http://hgvs.org/ mutnomen). Variant frequency was checked against ExAC to exclude frequent variants. The detected rare variants were matched with the Human Gene Mutation Database (HGMD) and ClinVar. For variant assessment, the in silico prediction programs MutationTaster, SIFT, and PolyPhen-2 as well as splice prediction tools implemented in the Alamut Software Suite (version 2.7_1, Interactive Biosoftware, Rouen, France), that is, Human Splicing Finder (version 3.1) and the NNSplice algorithm (http://www.fruitfly.org/seq_tools/splice.html), were used.

In correspondence to the guidelines of the American College of Medical Genetics and Genomics (ACMG) and the Association for Molecular Pathology (AMP) from 2015, 
detected variants were classified into the five ordinally scaled categories classes 1 to $5 .{ }^{29}$ After initial classification using in silico filtering programs and gene databases as described (phase 1 variant assessment), variants were classified in a second step with respect to clinical phenotype, segregation analysis, literature, in vitro data, and animal models (phase 2 variant assessment).

\section{Ethical Considerations}

Gene sequencing and processing of pseudonymized clinical and laboratory data were conducted at the Institute of Experimental Biomedicine, the University Children's Hospital, and the Institute of Human Genetics of the University of Würzburg between January 2015 and April 2016 in accordance with local Institutional Review Board guidelines and the declaration of Helsinki of 1964 with its later amendments. Written informed consent was obtained from each subject included.

\section{Results}

\section{Test System Validation}

Five DNA samples from patients with functionally characterized and genetically defined IPD served as controls during the validation process. Both sequencing procedure and data processing including in silico filtering exactly confirmed the known mutations and $\mathrm{CNV}$ in the five genes leading to distinct phenotypes: ADP receptor defect (heterozygous P2YR12 variant), leukocyte-adhesion deficiency type III (homozygous FERMT3 variant), MYH9-related macrothrombocytopenia (heterozygous MYH9 variant), TAR syndrome (hemizygous RBM8A noncoding intron 1 single nucleotide polymorphism [SNP] and heterozygous RBM8A microdeletion), and Wiskott-Aldrich syndrome (hemizygous WAS variant), respectively (- Supplementary Table S2).

\section{Gene Panel Analysis}

Three out of 38 datasets (cases 21-23) from patients with a so far unidentified genetic cause failed quality control and had thus to be excluded from further analysis. In total, 35 samples reached a mean 20 -fold coverage of $97.5 \%$ and could be analyzed as described ( - Fig. 1). Fifteen out of the 35 DNA samples $(43 \%)$ revealed one or more genetic variants in the tested 59 genes that could be classified as class 3 or higher following in silico assessment (phase 1 variant assessment; -Fig. 1). After phase 1 variant assessment, five variants (14\%) in the five distinct genes-HPS4 (case 10), AP3B1 (case 12), MYH9 (case 29), TUBB1 (case 31), and MPL (case 36)-were attributed to class 4 or 5 (-Fig. 1; - Table $\mathbf{1}$ ). Ten patients (29\%) carried 13 distinct class 3 variants in the genes HPS6 (case 1), ANKRD26 (case 2), ACTN1 (cases 7 and 27), TUBB1 (case 9), MYH9 (case 14), TBXA2R (case 16), GFI1B (cases 24 and 32), HPS1 (case 25), and FLNA and TPM4 (both case 32; -Fig. 1; -Table 1).

No further variant evaluation was required when patients carried class 4 or 5 variants (-Fig. 1; - Table $\mathbf{1}$ ). Class 4 or 5 variants in two genes associated with HPS (AP3B1 and HPS4) correlated well with the clinical phenotype of the two children (cases 10 and 12). ${ }^{30} \mathrm{~A}$ 1-month-old infant (case 29) and a 66-year-old woman (case 31) with congenital thrombocytopenia were diagnosed with a MYH9- or TUBB1-related disorder, respectively. ${ }^{31,32}$ The known homozygous MPL mutation, confirmed by Sanger sequencing, in a 7-month-old infant with severe thrombocytopenia and complete lack of megakaryocytes in the bone marrow aspirate (case 36) provided proof of congenital amegakaryocytic thrombocytopenia. $^{33}$

In case of class 3 variants, supplementary clinical and experimental evaluation was needed to further classify the detected variants (phase 2 variant assessment; -Fig. 1; - Table 1). Additional functional assays and segregation analysis provided evidence that the novel homozygous frameshift variant in the HPS6 gene of a 17-year-old adolescent (case 1 ) is causative for the clinical phenotype of HPS and was thus considered as class $4 .{ }^{34}$ Comprehensive genetic investigation was necessary to clear up the pathogenesis of a gray platelet-like syndrome in a family with life-threatening bleeding diathesis (case 24), ending up in the first description of an autosomal recessive GFI1B nonsense mutation in the alternatively spliced exon 9 , which selectively leads to severe macrothrombocytopenia and platelet dysfunction without critically impairing erythropoiesis. ${ }^{7}$ Two other adult patients with familial thrombocytopenia (cases 14 and 27) showed variants in the genes MYH9 and ACTN1 that are associated with thrombocytopenia due to impaired megakaryocytic and platelet cytoskeleton regulation according to previously described single reports. ${ }^{35-37}$ As a consequence, each of these four mentioned variants (HPS6, GFI1B, MYH9, and ACTN1) could be classified as class 4 variants after phase 2 variant assessment (-Fig. 1; - Table 1 ). If compound heterozygosity of the two heterozygous variants in the HPS1 gene in a patient with suspected HPS (case 25) is proven to be in trans-position by evaluating both currently unavailable parents, the detected heterozygous variants in combination would also be classified as class 4 (- Table $\mathbf{1}) .^{38}$

In one case of highly suggestive autosomal dominant thrombocytopenia (case 2), the identified but so far undescribed heterozygous variant outside the 5'UTR of the ANKRD26 gene did not segregate with the phenotype in the family and had thus to be classified as class 2 ( $~$ Table 1). Since clinical significance of novel heterozygous variants in ACTN1 and TBXA2R (cases 7 and 16) has not yet been completely evaluated, they remained categorized as class 3 variants (-Table 1). The heterozygous Q43P substitution in $\beta 1$-tubulin (case 9) was originally described to modulate platelet function and structure, but is better considered a modifier of platelet function due to its high MAF. ${ }^{39,40}$ In consequence, we have now classified this variant as class 2 .

Further evaluation becomes more complicated when the clinical phenotype is atypical due to variants in two or more genes: one child (case 32) with moderate thrombocytopenia, skin bleeding tendency, and positive family history (father with moderate thrombocytopenia and recurrent epistaxis; mother with postoperative bleeding and suspected thrombocytopathy) carried a class 3 variant in each of the GFI1B, TPM4, and FLNA genes (-Table $\mathbf{1}$ ). Cosegregation analysis in 
this family attributed the autosomal GFI1B and TPM4 variants to the affected father, while the X-chromosomal FLNA variant cosegregated with the non-syndromic mother. We reclassified this variant as class 2 (-Table 1). Overall, 8 of the 15 variants (53\%) classified as classes 3 to 5 after phase 2 assessment were novel (-Fig. 1).

After phase 2 variant assessment, 22 out of the 35 samples (63\%) did not provide any classes 3 to 5 variant in the investigated genes represented in the panel design ( - Fig. 1). Of these 22 samples, 10 were from patients with thrombocytopenia and the other 12 from patients with functionally suggestive thrombocytopathy (-Table 1 ). None of the suspected aspirin-like defects (cases 17 and 18) and none of the cases with delta-storage pool deficiency (cases 33, 37, and 38 ) showed any likely causative variant in the panel genes (-Table 1). For all these unexplained cases, an extended genetic analysis by WES or even whole genome sequencing (WGS) may uncover causative variants in novel genes not yet associated with IPD or variants in noncoding sequences (e.g., introns or regulatory elements) that are responsible for the pathogenesis (-Fig. 1).

\section{Discussion}

\section{Potentials in Patient Care and Clinical Research}

Our targeted, panel-based NGS approach was sensitive enough to confirm the variants of the five control patients (-Supplementary Table S2) including a complete heterozygous loss of the RBM8A gene, which is causative for TAR syndrome in a compound-heterozygous state with the predisposing SNP in RBM8A. ${ }^{41,42}$ In our study cohort of 35 investigated individuals with suspected but so far genetically undefined quantitative or qualitative IPD, we discovered 15 classes 3 to 5 variants ( $\mathbf{- F i g}$. 1), that may explain the corresponding clinical phenotype, in genes encoding transcription factors $(n=2)$, cytoskeletal proteins $(n=6)$, membrane receptors $(n=2)$, and intracellular vesicle transport regulators $(n=5)$.

Assuming that the two heterozygous variants in the HPS1 gene are in trans-position (case 25), each of the patient suffering from HPS in our cohort had a class 4 or 5 variant in one of the ten HPS genes. While in the group of 19 patients with isolated or combined thrombocytopenia nine patients (47\%) had one or more variants in platelet-associated genes, no variant was detected in the group of 12 patients with solitary, mild to moderate platelet function disorder other than HPS (-Table 1). This observation may reflect that we currently do not have sufficient knowledge about all genes involved in platelet function. Of note, some of the bleeding anomalies may be caused by vessel abnormalities or transient interfering factors and are thus not of platelet origin. Finally, we were able to attribute a molecular genetic diagnosis to 9 of the 35 investigated patients (26\%) after phase 2 variant assessment (-Fig. 1). Overall, the detection rates for known or novel variants in our targeted approach were similar to those recently published by the Scandinavian group. ${ }^{23}$

In comparison to sequential exon-by-exon, gene-by-gene Sanger sequencing, HTS methods may improve patient care as comorbidities are diagnosed earlier within regular screening programs: pulmonary fibrosis due to variants in HPS1, HPS4, or $A P 3 B 1^{43}$; differential impact on platelet count in TAR syndrome ${ }^{44}$; genotype-phenotype associations in MYH9- or DIAPH1-related disorders, such as hearing loss, cataract, or renal pathology 45,46 ; or risk of developing leukemia when predisposing genes, such as ANKRD26, ETV6, or RUNX1, are affected. ${ }^{2,47}$ In consequence, we consider that it is rational to sequence multiple genes associated with a particular phenotype simultaneously, for example, in case of thrombocytopenia or HPS. ${ }^{2,43}$

\section{Quality Assurance and Pitfalls}

HTS methods themselves and the flood of provided genetic data mask several risks or pitfalls: poor coverage of exon 1; false-positive or, to a minor extent, false-negative results during data processing; non-standardized variant classification; or insufficient evaluation of class 3 variants in routine diagnostics. In 2013, the ACMG sets professional clinical laboratory standards for NGS to assure high quality for each step from test development over sample processing to reporting criteria, ${ }^{48}$ followed by consensus guidelines of several European societies of human genetics. ${ }^{49-51}$

Newly established NGS systems must fulfill professional quality standards including assessment of the average read count, the uniformity of coverage, the allelic read percentage, strand biases, quality scores, and types of variants during validation and diagnostic processes. ${ }^{48,52}$ In our current panel, only $2.5 \%$ of the target sequences had a coverage less than 20-fold. To increase sensitivity of the assay in diagnostic settings, Sanger sequencing is still required to fill gaps in regions with low coverage, especially in GC-rich regions, which are often found in exon 1 . Novel techniques aiming to enhance enrichment in these areas may resolve this dilemma to a certain degree. WGS can bypass this problem, but the efforts for data analysis are much higher than for targeted or WES techniques. ${ }^{53}$ Besides, NGS approaches may fail to detect $\mathrm{CNV}$, epigenetic phenomena, or mosaicism; so, documentation of quality metrics (e.g., the fraction of 20 -fold coverage) is indispensable. ${ }^{52}$ Analyses must be repeated when quality parameters have failed (e. g., due to interfering substances or DNA sample impurity as we have encountered in 3 out of 43 samples; - Fig. 1).

A fundamental element of genetic testing is the interpretation of the detected variants. Several population-based (e.g., ExAC, dbSNP, dbVar, 1,000 genomes), disease-specific (e.g., ClinVar, OMIM, HGMD) or sequence-focused (e.g., NCBI Genome, RefSeqGene) databases as well as in silico prediction programs (e.g., SIFT, PolyPhen-2, MutationTaster) serve as a reference for a standardized process of interpretation. In correspondence to the ACMG and AMP guidelines from 2015, variants are currently classified into five categories according to scoring rules that weigh up population-based, in silico prediction, functional, segregational, and de novo data. ${ }^{29}$ In particular, class 3 variants need further investigation by functional studies, segregation analyses, cell culture assays, or animal models to support the likelihood or unlikelihood that the variant is disease causing. Variants identified by HTS 
should only be reported in clinical settings for variants affecting established genes causing the specific phenotype of the suspected IPD, also coined "Tier 1 genes." ${ }^{54}$ In addition, genetic counselling of index patients and their relatives is strongly based on the knowledge of bona fide identified variants and their mode of inheritance.

The process of variant interpretation is complicated when databases are biased toward a certain ethnical background (e.g., a patient with Asian or Arabic background compared with a Caucasian-biased database) or toward pathogenicity due to the fact that class 1 or 2 variants are reported less frequently to databases, if at all, than class 4 or 5 variants. Furthermore, Freson and Turro evoked convincing concerns that these databases are contaminated with class 1 or 2 variants incorrectly labeled as pathogenic, a circumstance that may lead to a serious feedback loop and, thus, to deceptive care of patients and their relatives. ${ }^{54}$

\section{Prospective of High-Throughput Sequencing in Inherited Platelet Disorders}

Since the exact underlying defect cannot be identified in more than one-third of patients with confirmed platelet function abnormality and DNA sequencing costs have rapidly declined over recent years, HTS techniques open up new horizons in this field. ${ }^{12-14,16}$ In 2015 and 2016 respectively, the Swiss and German public health systems introduced HTS analysis to the list of permitted genetic analyses for diagnostic reasons. By grouping the so far known and relevant genes for suspected pathophysiological mechanisms in platelet biogenesis or function, it becomes possible to have totally or at least partially reimbursed analytical efforts to confirm suspected diagnoses and genetically classify suspected but so far undefined entities of these rare diseases. HTS techniques may also boost scientific activities in the field of IPD, when functional studies, cell culture assays, or animal models allow characterization of novel variants, genes, or pathways. Patients benefit from a notably growing knowledge of these rare hematological diseases, their modes of inheritance, or their particular genetic features, as we could report for one family with severe autosomal recessive gray platelet-like syndrome detected by our NGS panel due to an alternative splice variant in the GFI1B gene. ${ }^{7}$

The authors consider that the presented NGS gene panel is capable of expanding molecular genetic testing to patients who have failed standard approaches to functionally diagnose or to genetically define IPD according to the published guidelines. ${ }^{10,11,14}$ Our experiences and those of international initiatives have been implemented in the study design of the joint patient registry for IPD of the GTH and GPOH with respect to phenotyping by HPO, assurance of comprehensive functional testing, and biomaterial banking. We propose a selective proceeding within the diagnostic algorithm, dependent on the suspected group of IPD (compare Fig. 5 in the study of Andres et $\mathrm{al}^{26}$ ).

Conflict of Interest

None declared.

\section{Acknowledgments}

We gratefully thank the permanent committee "Pediatrics" of the Society for Thrombosis and Haemostasis Research (Gesellschaft für Thrombose- und Hämostaseforschung; GTH) for financial support to the THROMKIDplus study group.

\section{References}

1 Nurden AT, Nurden P. Congenital platelet disorders and understanding of platelet function. Br J Haematol 2014;165(02): 165-178

2 Balduini CL, Melazzini F, Pecci A. Inherited thrombocytopenias recent advances in clinical and molecular aspects. Platelets 2017; 28(01):3-13

3 Sivapalaratnam S, Collins J, Gomez K. Diagnosis of inherited bleeding disorders in the genomic era. Br J Haematol 2017;179 (03):363-376

4 Nurden AT, Freson K, Seligsohn U. Inherited platelet disorders. Haemophilia 2012;18(Suppl 4):154-160

5 Noris P, Perrotta S, Bottega R, et al. Clinical and laboratory features of 103 patients from 42 Italian families with inherited thrombocytopenia derived from the monoallelic Ala156Val mutation of GPIb $\alpha$ (Bolzano mutation). Haematologica 2012;97(01):82-88

6 Monteferrario D, Bolar NA, Marneth AE, et al. A dominantnegative GFI1B mutation in the gray platelet syndrome. $\mathrm{N}$ Engl J Med 2014;370(03):245-253

7 Schulze H, Schlagenhauf A, Manukjan G, et al. Recessive grey platelet-like syndrome with unaffected erythropoiesis in the absence of the splice isoform GFI1B-p37. Haematologica 2017; 102(09):e375-e378

8 Harrison P, Lordkipanidzé M. Clinical tests of platelet function. In: Platelets. 3rd ed. New York: Academic Press; 2013:519-545

9 Harrison P, Mackie I, Mumford A, et al; British Committee for Standards in Haematology. Guidelines for the laboratory investigation of heritable disorders of platelet function. $\mathrm{Br} \mathrm{J}$ Haematol 2011;155(01):30-44

10 Knöfler R, Eberl W, Schulze H, et al. [Diagnosis of inherited diseases of platelet function. Interdisciplinary S2K guideline of the Permanent Paediatric Committee of the Society of Thrombosis and Haemostasis Research (GTH e. V.)]. Hamostaseologie 2014;34(03):201-212

11 Gresele P; Subcommittee on Platelet Physiology of the International Society on Thrombosis and Hemostasis. Diagnosis of inherited platelet function disorders: guidance from the SSC of the ISTH. J Thromb Haemost 2015;13(02):314-322

12 Gresele P, Harrison P, Bury L, et al. Diagnosis of suspected inherited platelet function disorders: results of a worldwide survey. J Thromb Haemost 2014;12(09):1562-1569

13 Wetterstrand K DNA Sequencing Costs: Data from the NHGRI Genome Sequencing Program (GSP) Available at: www.genome. gov/sequencingcostsdata. Accessed July 1, 2018

14 Gresele P, Bury L, Falcinelli E. Inherited platelet function disorders: algorithms for phenotypic and genetic investigation. Semin Thromb Hemost 2016;42(03):292-305

15 Mannhalter C. [New developments in molecular biological diagnostic]. Hamostaseologie 2017;37(02):138-151

16 Lentaigne C, Freson K, Laffan MA, Turro E, Ouwehand WH; BRIDGE-BPD Consortium and the ThromboGenomics Consortium. Inherited platelet disorders: toward DNA-based diagnosis. Blood 2016;127(23):2814-2823

17 Simeoni I, Stephens JC, Hu F, et al. A high-throughput sequencing test for diagnosing inherited bleeding, thrombotic, and platelet disorders. Blood 2016;127(23):2791-2803

18 Bariana TK, Ouwehand WH, Guerrero JA, Gomez K; BRIDGE Bleeding, Thrombotic and Platelet Disorders and ThromboGenomics Consortia. Dawning of the age of genomics for platelet 
granule disorders: improving insight, diagnosis and management. Br J Haematol 2017;176(05):705-720

19 Westbury SK, Turro E, Greene D, et al; BRIDGE-BPD Consortium. Human phenotype ontology annotation and cluster analysis to unravel genetic defects in 707 cases with unexplained bleeding and platelet disorders. Genome Med 2015;7(01):36

20 Fletcher SJ, Johnson B, Lowe GC, et al; UK Genotyping and Phenotyping of Platelets Study Group. SLFN14 mutations underlie thrombocytopenia with excessive bleeding and platelet secretion defects. J Clin Invest 2015;125(09):3600-3605

21 Leo VC, Morgan NV, Bem D, et al; UK GAPP Study Group. Use of next-generation sequencing and candidate gene analysis to identify underlying defects in patients with inherited platelet function disorders. J Thromb Haemost 2015;13(04):643-650

22 Johnson B, Lowe GC, Futterer J, et al; UK GAPP Study Group. Whole exome sequencing identifies genetic variants in inherited thrombocytopenia with secondary qualitative function defects. Haematologica 2016;101(10):1170-1179

23 Leinøe E, Zetterberg E, Kinalis S, et al. Application of whole-exome sequencing to direct the specific functional testing and diagnosis of rare inherited bleeding disorders in patients from the Öresund Region, Scandinavia. Br J Haematol 2017;179(02):308-322

24 Bastida JM, Lozano ML, Benito R, et al. Introducing high-throughput sequencing into mainstream genetic diagnosis practice in inherited platelet disorders. Haematologica 2018;103(01):148-162

25 Althaus K, Najm J, Greinacher A. MYH9 related platelet disorders often unknown and misdiagnosed. Klin Padiatr 2011;223(03): $120-125$

26 Andres O, Henning K, Strauß G, Pflug A, Manukjan G, Schulze H. Diagnosis of platelet function disorders: a standardized, rational, and modular flow cytometric approach. Platelets 2018;29(04):347-356

27 Crazzolara R, Maurer K, Schulze H, Zieger B, Zustin J, Schulz AS. A new mutation in the KINDLIN-3 gene ablates integrin-dependent leukocyte, platelet, and osteoclast function in a patient with leukocyte adhesion deficiency-III. Pediatr Blood Cancer 2015;62 (09):1677-1679

28 Balduini CL, Pecci A, Noris P. Diagnosis and management of inherited thrombocytopenias. Semin Thromb Hemost 2013;39 (02):161-171

29 Richards S, Aziz N, Bale S, et al; ACMG Laboratory Quality Assurance Committee. Standards and guidelines for the interpretation of sequence variants: a joint consensus recommendation of the American College of Medical Genetics and Genomics and the Association for Molecular Pathology. Genet Med 2015;17(05):405-424

30 Fontana S, Parolini S, Vermi W, et al. Innate immunity defects in Hermansky-Pudlak type 2 syndrome. Blood 2006;107(12): 4857-4864

31 Kelley MJ, Jawien W, Ortel TL, Korczak JF. Mutation of MYH9, encoding non-muscle myosin heavy chain A, in May-Hegglin anomaly. Nat Genet 2000;26(01):106-108

32 Auer PL, Teumer A, Schick U, et al. Rare and low-frequency coding variants in CXCR2 and other genes are associated with hematological traits. Nat Genet 2014;46(06):629-634

33 van den Oudenrijn S, Bruin M, Folman CC, et al. Mutations in the thrombopoietin receptor, $\mathrm{Mpl}$, in children with congenital amegakaryocytic thrombocytopenia. Br J Haematol 2000;110(02):441-448

34 Andres O, Wiegering V, König EM, et al. A novel two-nucleotide deletion in HPS6 affects mepacrine uptake and platelet dense granule secretion in a family with Hermansky-Pudlak syndrome. Pediatr Blood Cancer 2017;64(05); doi: 10.1002/pbc.26320

35 Miyazaki K, Kunishima S, Fujii W, Higashihara M. Identification of three in-frame deletion mutations in MYH9 disorders suggesting an important hot spot for small rearrangements in MYH9 exon 24. Eur J Haematol 2009;83(03):230-234

36 Bottega R, Marconi C, Faleschini M, et al. ACTN1-related thrombocytopenia: identification of novel families for phenotypic characterization. Blood 2015;125(05):869-872
37 Kunishima S, Okuno Y, Yoshida K, et al. ACTN1 mutations cause congenital macrothrombocytopenia. Am J Hum Genet 2013;92 (03):431-438

38 Oh J, Bailin T, Fukai K, et al. Positional cloning of a gene for Hermansky-Pudlak syndrome, a disorder of cytoplasmic organelles. Nat Genet 1996;14(03):300-306

39 Freson K, De Vos R, Wittevrongel C, et al. The TUBB1 Q43P functional polymorphism reduces the risk of cardiovascular disease in men by modulating platelet function and structure. Blood 2005;106(07):2356-2362

40 Navarro-Núñez L, Lozano ML, Rivera J, et al. The association of the beta1-tubulin Q43P polymorphism with intracerebral hemorrhage in men. Haematologica 2007;92(04):513-518

41 Klopocki E, Schulze H, Strauss G, et al. Complex inheritance pattern resembling autosomal recessive inheritance involving a microdeletion in thrombocytopenia-absent radius syndrome. Am J Hum Genet 2007;80(02):232-240

42 Albers CA, Paul DS, Schulze H, et al. Compound inheritance of a low-frequency regulatory SNP and a rare null mutation in exonjunction complex subunit RBM8A causes TAR syndrome. Nat Genet 2012;44(04):435-439, S1-S2

43 Huizing M, Malicdan MCV, Gochuico BR, et al. Hermansky-Pudlak Syndrome. In: GeneReviews((R)). Seattle, WA: University of Washington; 1993-2018

44 Manukjan G, Bösing H, Schmugge M, Strauß G, Schulze H. Impact of genetic variants on haematopoiesis in patients with thrombocytopenia absent radii (TAR) syndrome. Br J Haematol 2017;179 (04):606-617

45 Pecci A, Ma X, Savoia A, Adelstein RS. MYH9: structure, functions and role of non-muscle myosin IIA in human disease. Gene 2018; 664:152-167

46 Stritt S, Nurden P, Turro E, et al; BRIDGE-BPD Consortium. A gain-of-function variant in DIAPH1 causes dominant macrothrombocytopenia and hearing loss. Blood 2016;127(23): 2903-2914

47 Melazzini F, Palombo F, Balduini A, et al. Clinical and pathogenic features of ETV6-related thrombocytopenia with predisposition to acute lymphoblastic leukemia. Haematologica 2016;101(11): 1333-1342

48 Rehm HL, Bale SJ, Bayrak-Toydemir P, et al; Working Group of the American College of Medical Genetics and Genomics Laboratory Quality Assurance Committee. ACMG clinical laboratory standards for next-generation sequencing. Genet Med 2013;15(09): 733-747

49 Schweizerisches Konsensusdokument der Schweizerischer Gesellschaft für Medizinische Genetik (SGMG). Bonnes pratiques - Für die klinische Anwendung der Hochdurchsatz-Sequenzierung (HDS). Schweizerische Gesellschaft für Medizinische Genetik; Version 1, 2014. Available at: http://sgmg.ch/wordpress/wp-content/uploads/2015/12/Bonnes_Pratiques_BAG.pdf

50 Matthijs G, Souche E, Alders M, et al; EuroGentest; European Society of Human Genetics. Guidelines for diagnostic next-generation sequencing. Eur J Hum Genet 2016;24(01):2-5

51 Deutsche Gesellschaft für Humangenetik. S1 Leitlinie: Molekulargenetische Diagnostik mit Hochdurchsatz-Verfahren der Keimbahn, beispielsweise mit Next-Generation Sequencing. medgen 2018;30(02):278-292, AWMF Register-Nr. 078/016. Available at: https://doi.org/10.1007/s11825-018-0189-z

52 Gargis AS, Kalman L, Berry MW, et al. Assuring the quality of nextgeneration sequencing in clinical laboratory practice. Nat Biotechnol 2012;30(11):1033-1036

53 Tilak MK, Botero-Castro F, Galtier N, Nabholz B. Illumina library preparation for sequencing the GC-rich fraction of heterogeneous genomic DNA. Genome Biol Evol 2018;10(02):616-622

54 Freson K, Turro E. High-throughput sequencing approaches for diagnosing hereditary bleeding and platelet disorders. J Thromb Haemost 2017;15(07):1262-1272 